\title{
Filter Banks for Next Generation Multicarrier Wireless Communications
}

\author{
Markku Renfors (EURASIP Member), ${ }^{1}$ Pierre Siohan, ${ }^{2}$ \\ Behrouz Farhang-Boroujeny, ${ }^{3}$ and Faouzi Bader ${ }^{4}$ \\ ${ }^{1}$ Department of Communications Engineering, Tampere University of Technology, P.O. Box 553, 33101 Tampere, Finland \\ ${ }^{2}$ Orange Labs, France Telécom, 4 Rue du Clos Courtel, B.P. 91226, 35512 Cesson Sévigné Cedex, France \\ ${ }^{3}$ Department of Electrical and Computer Engineering, University of Utah, Salt Lake City, UT 84112-9206, USA \\ ${ }^{4}$ Centre Tecnologic de Telecommunication de Catalunya (CTTC), Parc Mediterrani de la Tecnologia, \\ Avinguda del Canal Olimpic, Casstelldefels, 08860 Barcelona, Spain
}

Correspondence should be addressed to Markku Renfors, markku.renfors@tut.fi

Received 3 May 2010; Accepted 3 May 2010

Copyright (c) 2010 Markku Renfors et al. This is an open access article distributed under the Creative Commons Attribution License, which permits unrestricted use, distribution, and reproduction in any medium, provided the original work is properly cited.

The theoretical capacity limits in communications can be approached by multicarrier techniques. With radio channels, multicarrier techniques can be combined with multiantenna transmitters and receivers to provide efficiency. Existing or planned transmission systems rely on the OFDM technique to reach these goals and a considerable amount of research has been devoted to these techniques during the last 20 years. However OFDM has a number of drawbacks, such as the use of the cyclic prefix to cope with the channel impulse response which results in a loss of capacity and the requirement of block processing to maintain orthogonality among all the subcarriers. Furthermore, the leakage among frequency subbands has a serious impact on the performance of FFT-based spectrum sensing and OFDM-based cognitive radio in general.

On the other hand, digital filter banks find various good applications in communications signal processing. In general, they can be used to obtain very sharp frequency selectivity to isolate different communications frequency channels from each other and from interfering spectral components. This can be done in a very flexible and dynamic manner. Thus filter banks constitute a very powerful generic tool for software-defined radios and spectrally agile communication systems.

So far, some attempts have been made to introduce filter bank multicarrier (FBMC) in the radio communications arena, through proprietary schemes, in particular the IOTA
(Isotropic Orthogonal Transform Algorithm). However, the full exploitation and optimization of FBMC techniques in the context of radio evolution, such as dynamic access, as well as their combination with MIMO techniques, have not been considered sufficiently.

This special issue aims to report advances in these communication aspects of FBMC, helping to make full use of FBMC as a new physical layer for future radio communication systems. We received 18 submission altogether, out of which ten were accepted through a peer review process.

The first paper, "Cosine modulated and offset QAM filter bank multicarrier techniques: a continuous-time prospect" authored by B. Farhang-Boroujeny and C. H. (George) Yuen, presents a tutorial review relating the classical works on FBMC systems, developed prior of the era of OFDM, to the main filter bank design approaches used today for FBMC systems. The paper also reviews the recent novel developments in the design of FBMC systems that are tuned to cope with fast fading wireless channels.

N. Moret and A. M. Tonello address the efficient realization of a filtered multitone (FMT) modulation system in the second paper entitled "Design of orthogonal filtered multitone modulation systems and comparison among efficient realizations". The paper analyzes three different realization structures, presenting also numerical comparisons, and compares the best FMT approach with a cyclically prefixed OFDM system in the IEEE 802.11 wireless LAN channel. 
The third paper, "Optimized paraunitary filter banks for time-frequency channel diagonalization" by Z. Ju et al. develops a method to diagonalize a doubly dispersive channel in the time-frequency domain using a filter bank approach. The related paraunitary filter bank design problem is formulated as a convex optimization problem, and the performance of the resulting window is investigated under different channel conditions.

The fourth paper, "Spectral efficiency comparison of OFDM/FBMC for uplink cognitive radio networks" by $\mathrm{H}$. Zhang et al. studies channel capacity of cognitive radio (CR) networks using CP-OFDM and FBMC waveforms, taking into consideration the effects of resource allocation algorithms, intercell interference due to timing offsets, and Rayleigh fading. Final results show that FBMC can achieve higher channel capacity than OFDM because of the low spectral leakage of its prototype filter.

M. Shaat and F. Bader address the problem of resource allocation in multicarrier-based CR networks in the fifth paper entitled "Computationally efficient power allocation algorithm in multicarrier-based cognitive radio networks: OFDM and FBMC systems". The objective is to maximize the downlink capacity of the network under constraints on both total power and interference introduced to the primary users. The performance of the proposed low-complexity algorithm is investigated for OFDM- and FBMC-based CR systems.

In the sixth paper, "Packet format design and decision directed tracking methods for filter bank multicarrier systems", P. Amini and B. Farhang-Boroujeny develop a packet format for FBMC systems together with algorithms for carrier frequency and timing recovery. Also methods for channel estimation as well as carrier and timing tracking loops are proposed.

In the seventh paper, entitled "Joint symbol timing and CFO estimation for OFDM/OQAM systems in multipath channels", T. Fusco et al. develop different maximumlikelihood based approaches for estimating carrier-frequency offsets and symbol timing offsets using short preambles in the FBMC transmission bursts. Good performance for a lowcomplexity approximate ML estimator is demonstrated.

The eighth paper, "Pilot-based synchronization and equalization in filter bank multicarrier communications" authored by T. H. Stitz et al., presents a detailed analysis of synchronization and channel estimation methods for FBMC based on scattered pilots. The special problems related to using scattered pilot-based schemes in FBMC are highlighted. The channel parameter estimation and compensation are successfully performed totally in the frequency domain, in a subchannel-wise fashion, which is appealing in spectrally agile and cognitive radio scenarios.

The ninth paper is entitled "Decoding schemes for FBMC with single-delay STTC" and authored by C. Lélé and D. Le Ruyet. The paper develops space-time trellis coding schemes for FBMC, addressing the challenge that the OQAM signal model of FBMC makes the decoding process more challenging compared to the CP-OFDM case. The developed iterative decoding scheme for FBMC is shown to slightly outperform CP-OFDM.
The tenth paper, "The Alamouti scheme with CDMAOFDM/OQAM" by C. Lélé et al. introduces first the fact that the well-known Alamouti decoding scheme cannot be simply combined with the OQAM subcarrier modulation scheme of FBMC. The paper then develops Alamouti coding schemes by combining CDMA component with OFDM/OQAM.

We would like to thank all authors for their contributions to our special issue, the reviewers for their help in selecting papers, and the Editor-in-Chief Phillip Regalia and the Editorial Office of the Journal for their support.

\author{
Markku Renfors \\ Pierre Siohan \\ Behrouz Farhang-Boroujeny \\ Faouzi Bader
}

